\title{
Grid Frequency Support by Single-Phase Electric Vehicles Employing an Innovative Virtual Inertia Controller
}

\author{
Rezkalla, Michel M.N.; Zecchino, Antonio; Pertl, Michael; Marinelli, Mattia
}

Published in:

Proceedings of the 51st International Universities Power Engineering Conference

Link to article, DOI:

10.1109/UPEC.2016.8113980

Publication date:

2016

Document Version

Peer reviewed version

Link back to DTU Orbit

\section{Citation (APA):}

Rezkalla, M. M. N., Zecchino, A., Pertl, M., \& Marinelli, M. (2016). Grid Frequency Support by Single-Phase Electric Vehicles Employing an Innovative Virtual Inertia Controller. In Proceedings of the 51st International Universities Power Engineering Conference IEEE. https://doi.org/10.1109/UPEC.2016.8113980

\section{General rights}

Copyright and moral rights for the publications made accessible in the public portal are retained by the authors and/or other copyright owners and it is a condition of accessing publications that users recognise and abide by the legal requirements associated with these rights.

- Users may download and print one copy of any publication from the public portal for the purpose of private study or research.

- You may not further distribute the material or use it for any profit-making activity or commercial gain

- You may freely distribute the URL identifying the publication in the public portal 


\title{
Grid Frequency Support by Single-Phase Electric Vehicles Employing an Innovative Virtual Inertia Controller
}

\author{
Michel Rezkalla, Antonio Zecchino, Michael Pertl, Mattia Marinelli \\ Center for Electric Power and Energy, Department of Electrical Engineering, DTU - Technical University of Denmark \\ Contact person: Michel Rezkalla (mirez@elektro.dtu.dk)
}

\begin{abstract}
The displacement of conventional generation by converter connected resources reduces the available rotational inertia in the power system, which leads to faster frequency dynamics and consequently a less stable frequency behavior. Virtual inertia, employing energy storage systems, could be used to limit the rate of change of frequency of power systems, thus, improving frequency dynamics. Electric vehicles (EVs) can represent a reliable solution to enhance frequency stability due to their fast response and capability to provide a large amount of aggregated power. On one hand, EVs are capable of adjusting the battery charging process (i.e., power flow) according to pre-defined algorithms. On the other hand, in case of islanded operation (i.e., low inertia), some of the EV's technical constraints might cause oscillations. This study presents two control algorithms which show that the EVs are capable of providing virtual inertia support. The first controller employs a traditional droop control, while the second one is equipped with an innovative control algorithm to eliminate likely oscillations. It is shown that, the proposed innovative control algorithm compared to the traditional droop control, assures same effects in terms of frequency but reducing significantly the number of variation of the EV's current set-point.
\end{abstract}

Index Terms-Electric Vehicle, Virtual Inertia, Grid Inertia, Time Domain Simulation, Converter Connected Resources

\section{INTRODUCTION}

In conventional power systems, the electricity generation is based on rotating synchronous machines. The grid frequency is maintained within an admissible range all the time to guarantee a secure and stable operation. Following a large disturbance that causes the frequency to significantly deviate from its nominal value, the synchronous generators (SGs) inherently release the kinetic energy stored in their rotating masses, and the SGs that have operating reserve increase their active power via primary and secondary controls [1].

Traditionally, inertial response has not been considered as an ancillary service, but rather as a natural characteristic of the power system. Due to the high integration of converter connected resources, replacing conventional generation, several transmission system operators in different countries began to recognize the value of inertial response by wind power plants [2]-[5].

Different impacts on the primary frequency control due to the increased penetration of renewable energy are presented in [6]. The lower system inertia is one of those impacts that would increase the requirements for primary frequency control reserves in order to arrest frequency at the same nadir (i.e., lowest frequency reached) following the sudden loss of generation.

The growing number of Electric Vehicles (EVs) is typically seen as an additional load on the grid from system operators perspective [7], [8]. However, EVs are also one of the imminent candidates for providing grid regulation services (i.e., frequency and voltage control), since most of the time they are plugged into a charging spost. In principle, they are able to provide fastregulating power in both directions in case of Vehicle-to-Grid (V2G), or just to modulate the charging power [9]. Nevertheless, EV's technical characteristics arise different challenges such as: the limited energy capacity of each $\mathrm{EV}$, the requested activation time (i.e., the time of full transition response of the active power from the moment of activation of the controller) [10]. The EV's limited energy capacity could be solved by aggregating a large number of EVs, while the time delay issue could be solved employing high quality measurement devices to reduce measurement time and therefore reducing the overall response time.

In this study, the modeled EVs are not equipped with V2G capability. To comply with the IEC 61851 standard, they are capable of modulating the charging current between 6 and 16 A with 1-A steps [11], [12]. This technical constraint might cause the EV's current to oscillate.

This study presents the EV's capability of providing frequency support by employing a virtual inertia controller. Two different controllers are implemented and investigated, virtual inertia controller and virtual inertia controller integrated with a stabilizer algorithm. A comparative analysis between the two controller is conducted. Moreover, a sensitivity analysis is carried out, to emphasise the EV's time delay effect on the frequency stability.

In order to test the EVs capability of providing virtual inertia, an islanded microgrid is modeled. The modeled grid is a reproduction of an islanded configuration of the experimental low voltage grid SYSLAB PowerLabDK research infrastructure, which is located at the DTU Ris $\emptyset$ campus. Both, controllers and the analysed grid are implemented in the power system 
simulation software PowerFactory DIgSILENT.

This paper is divided into four sections: Section II presents the specifications of the two controllers and the analysed scenarios. Section III presents the results of the comparison between the controllers and the time delay influence on the virtual inertia controller. In section IV conclusion and future work are reported.

\section{Controller Specification And Study CASES}

Virtual inertia in the power system could be emulated if the active power delivered (or absorbed) through the converter of an energy storage system is controlled in inverse proportion to the derivative of the grid frequency $(d f / d t)$.

This section presents the specifications of the two controllers, the network model and the different scenarios.

\section{A. Controllers specifications}

In this study, two controllers are implemented and investigated, namely virtual inertia controller (VIC) and virtual inertia controller integrated with a stabilizer algorithm (VIC_S). Both controllers use single-phase electric vehicles as an energy source, taking advantages of the installed batteries, by modulating the charging current.

The controllers' participation is provided by a droop control, which represents how much the controllers are sensible to the rate of change of frequency (ROCOF). In this study three different droops have been considered: $\alpha$ (the ROCOF limits are $\pm 0.0625 \mathrm{~Hz} / \mathrm{s}), \beta( \pm 0.125 \mathrm{~Hz} / \mathrm{s})$ and $\gamma( \pm 0.1875$ $\mathrm{Hz} / \mathrm{s})$. The three droops are ROCOF-Current droops and are presented in Fig. 1. The solid lines represent the 1-A steps function required by the IEC 61851 standard [11].

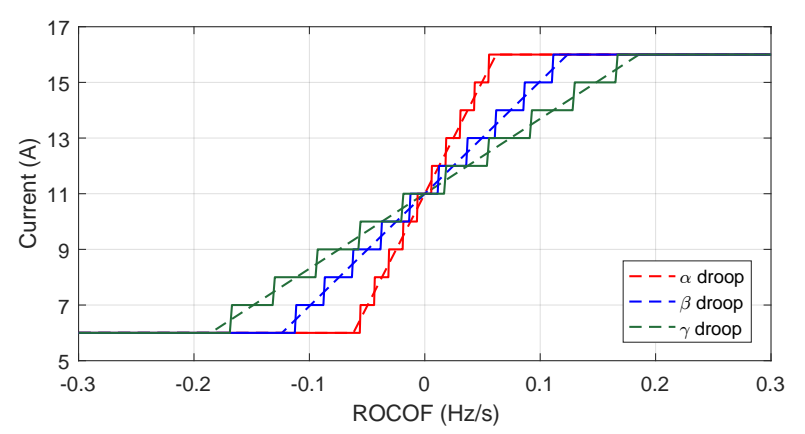

Fig. 1. $\alpha, \beta$ and $\gamma$ ROCOF-Current droops: ideal functions (solid lines) and step functions (dashed lines).

The two controllers are implemented in PowerFactory and the block diagram is shown in Fig. 2. It consists of three main blocks as shown in Fig. 2_A: The frequency measurement device, the control algorithm and the EV model. The control algorithm block diagram is presented in Fig. 2_B. The green blocks and arrows are not needed in case of VIC.

1) Virtual Inertia Controller (VIC): The VIC is based on a traditional droop control sensitive to the ROCOF, and no dead-band has been considered. It calculates the ROCOF, and according to a predefined droop, it changes the EV's current set-point.
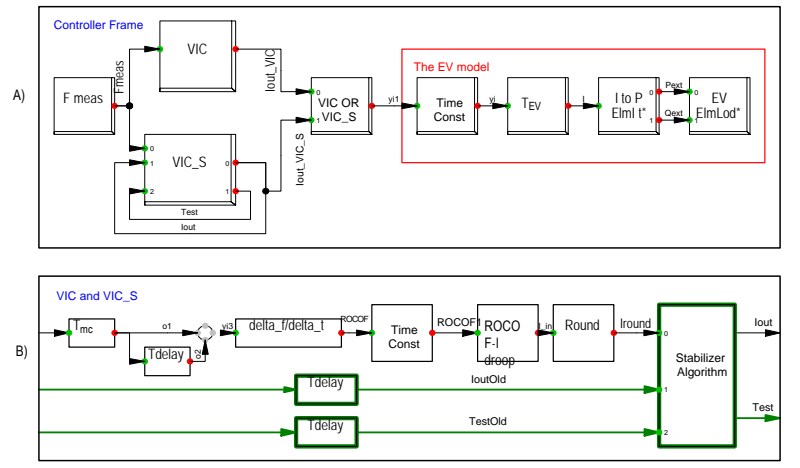

Fig. 2. Controller block diagram. The highlights show the parts added for VIC_S. (A) shows the measurement block, the control algorithm (VIC or VIC_S) and the EV model. (B) shows the control algorithm block diagram.

The VIC controller is composed by:

- A time delay block to represent the digital time delay due to measurement and communication, called $T_{m c}$.

- A ROCOF calculation block.

- A time constant block as low-pass filter.

- A block with the ROCOF-Current droop.

- A round function block to recreate steps of $1 \mathrm{~A}$.

The EV model used by the two controllers is composed by:

- A time constant block to model the EV battery dynamics.

- A time delay block to represent the delay due to internal EV communication and activation of the inverter, called $T_{E V}$.

- A block that converts the current to a power signal.

- A load block representing the EV.

2) Virtual Inertia Controller Stabilizer (VIC_S): The VIC_S controller is composed mainly by the VIC integrated with a stabilizing algorithm. The controller block diagram is shown in Fig. 2_B.

As mentioned before, the modeled EVs respect the technical constraint of changing the current set-point only in 1-A steps. To comply with the mentioned limitation, the round block rounds the current set-point calculated as function of the ROCOF. This might cause 1-A oscillations, especially in presence of steep droops, low inertia grid, large response times and high share of EV's power employed as reserve. The reason is, if the calculated current set-point is close to the midpoint between two consecutive set-points, it may be continuously rounded up and down.

For example, if the calculated current is $7.51 \mathrm{~A}$, then the set-point will be $8 \mathrm{~A}$. The same set-point signal is sent to an aggregated number of EVs. The difference between the required $7.51 \mathrm{~A}$ and the actual $8 \mathrm{~A}$ in all the EVs would cause a significant change in the power flow in terms of total absorbed active power. This will affect the frequency trend, resulting in a new calculated current around 7.49 A, rounded down to $7 \mathrm{~A}$. This process will turn in a loop that determines the 1 A-oscillations.

The aim of the stabilizer algorithm is to avoid 1-A oscillations while allowing larger but less-probable ones (e.g., 2-A 
oscillations). This will reduce the overall probability of current oscillations.

The stabilizer algorithm flow-chart is presented in Fig. 3.

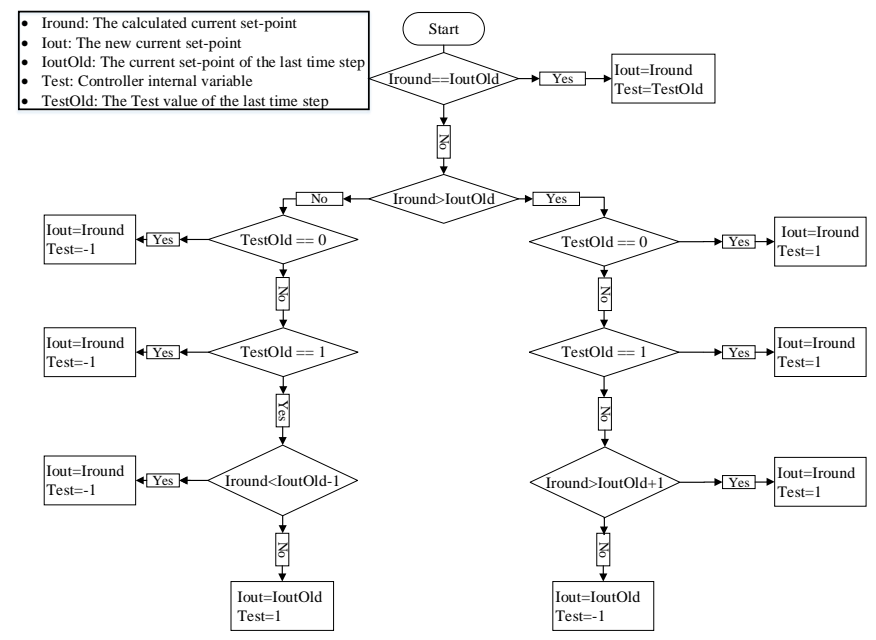

Fig. 3. Stabilizer Algorithm Flow-Chart of the VIC_S

The controller calculates the current set-point Iout based on the stabilizer algorithm which evaluates two conditions: The current set-point Iout and an internal parameter Test.

The first condition is obtained by comparing the new calculated current set-point Iround with the one from the previous time step IoutOld. The second condition is evaluated through a consideration of a memory status TestOld, which is the Test from the previous time step. Test indicates whether or not, and how, the current set-point is going to change compared to the value of previous time step. It will take the values of $-1,0$ or 1 . The -1 indicates that, the current set-point has been reduced in the previous time step, 1 that it has been increased, while 0 is utilized for the initialization of the controller.

Since the aim of the controller is to avoid 1-A oscillations, the algorithm prevents 1-A step from one time step to the next one under certain conditions. To do this, the algorithm compares Iround with IoutOld taking into account the value of TestOld. For instance, in case Iround is greater than IoutOld by 1 A difference, and TestOld is -1 then Iout will be kept as IoutOld. Iout will be changed only when the difference is at least 2 A up or 1 A down.

To give a practical example, if Iround is 9 A, IoutOld is 8 A and TestOld is -1 then the controller prevents the current change. In fact Iout will take the same value of IoutOld and Test will be kept as TestOld. In case Iround will increase to $10 \mathrm{~A}$ (or decrease to $7 \mathrm{~A}$ ), then the set-point change will be allowed: Iout will be $10 \mathrm{~A}$ (or $7 \mathrm{~A}$ ) and Test will be 1 (or -1).

\section{B. Network model}

This study has been carried out by means of root-meansquare (RMS) simulations activities in DIgSILENT PowerFactory software environment.

In order to allow a future practical experimental validation study, the modelled microgrid, has been built considering real available power system components. Specifically, the following devices have been considered for the proposed simulation studies:

- Three controllable EVs, each equipped with single-phase 16 A $(230 \mathrm{~V})$ charger and $24 \mathrm{kWh}$ Lithium-ion battery. The charging current can be modulated between 6 and 16 A with steps of 1 A to comply with the technical constraints imposed by the IEC 61851 standard [11]. The EVs initial current set-point is $11 \mathrm{~A}$, the central point, which assures room to increase and decrease the charging level equally.

- A 60 kVA diesel synchronous generator, with active power provision up to $48 \mathrm{~kW}$. It provides inertia to the microgrid. Since designed for operating in microgrid contexts, the inertia of the unit is relatively high, i.e., $2 \mathrm{H}=50 \mathrm{~s}$. The diesel's governor is activated to provide primary frequency control.

- A controllable $45 \mathrm{~kW}$ (i.e., $15 \mathrm{~kW}$ per phase, adjustable with steps of $0.1 \mathrm{~kW}$ ) resistive load unit.

- A $10 \mathrm{~kW}$ Aircon wind turbine equipped with full converter and active stall power control.

The modelled microgrid is composed by two bus-bars connected by a $725 \mathrm{~m}$ cable. The diesel generator and the wind turbine are connected at the first bus-bar, while the three EVs and the load are connected at the second one.

\section{Scenarios}

In order to test the EVs capability of providing virtual inertia support and to evaluate the effectiveness of the two controllers, three scenarios have been analyzed. In the three scenarios, the system response was triggered by changing the load consumption. The total absorbed active power is $19.5 \mathrm{~kW}$, split into $12 \mathrm{~kW}$ from the load unit and $7.5 \mathrm{~kW}$ from the three EVs $(3 * 2.5 \mathrm{~kW})$. In the first two scenarios, the total absorbed active power is delivered only by the diesel generator. In the third scenario the active power is delivered also by the Aircon wind turbine.

The purpose of the first scenario is to emphasize the time delay effect on the VIC. The load event takes place at zero seconds of the RMS simulation by a $25 \%(3 \mathrm{~kW})$ increase of the load consumption. It represents around $15 \%$ increase of the total generated active power and around $5 \%$ of the diesel rated power. The choice of this large load step has been done to compensate the large inertia. The system has been analysed employing the $\beta$ droop and applying the following time delays: $2,3.5$ and $5.5 \mathrm{~s}$. The considered time delay represents the total response time of the vehicle and the controllers to the detected frequency deviation (i.e., $T_{E V}+T_{m c}$ ).

The $5.5 \mathrm{~s}$ is chosen as maximum acceptable time delay in accordance with the experimental results presented in [10]. In [12], the authors claim that most of the times the total time delay is between 2 to $3 \mathrm{~s}$. For this reason, in the following analysis, $2 \mathrm{~s}$ is assumed to be the lowest time delay. The $3.5 \mathrm{~s}$ is chosen as intermediate value.

The second scenario aims at providing a general evaluation and comparison between VIC and VIC_S in case of contingen- 
cies. To better evaluate the two controllers, the system response is analysed employing the three different droops, namely $\alpha, \beta$ and $\gamma$, adapting as time delay $2 \mathrm{~s}$. The same load event was initiated to evaluate the behavior of the controller.

In the third scenario, the Aircon wind turbine is connected to the analysed microgrid. It originates continuous fluctuations of power generation and consequently less stable frequency behaviour. This made it possible to evaluate the efficiency of the two controllers in a more realistic situation, where frequency is always fluctuating. A 10 minutes wind production profile, in terms of active and reactive power, has been considered and reported in Fig. 4.

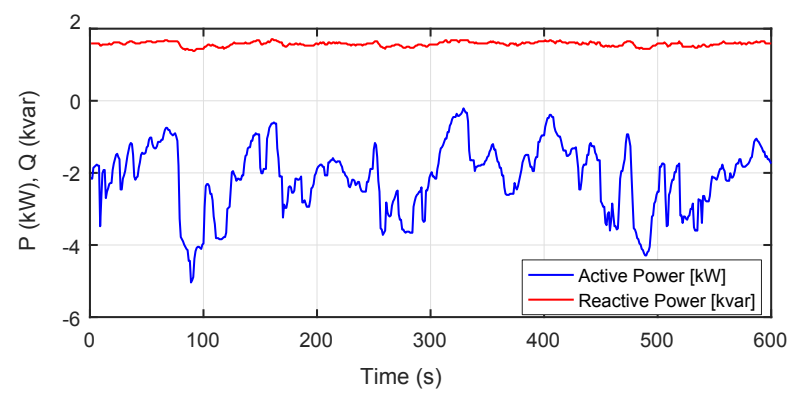

Fig. 4. 10-minutes active and reactive power wind generation profile.

In this case, the load events include load-increase and loaddecrease, so that both over and under frequency dynamics can be analysed. Four load events have been applied, by changing the load consumption by $25 \%$ of it's rated power as flows, by $+25 \%,-25 \%,-25 \%$ and $+25 \%$, respectively at, $0 \mathrm{~s}, 180 \mathrm{~s}, 360$ $\mathrm{s}$ and $540 \mathrm{~s}$.

The third scenario is divided into two study cases, the first study case reports a comparison between the two controllers employing the three droops. The second one reports the improvements and advantages of applying a combined droop in case of VIC_S, specifically combining the $\alpha$ and $\beta$ droops.

\section{RESULTS}

This section is composed by three subsections, which present the results of the three scenarios.

\section{A. Scenario 1: Sensitivity analysis of the time delay}

The first scenario presents the time delay effect on the controller and consequently on the frequency behaviour. The frequency and the ROCOF trends (signal "ROCOF1" in Fig. 2_B) are presented in Fig. 5_A and Fig. 5_B, respectively.

Fig. 5 shows that the frequency starts to have undesired behaviour by adapting $5.5 \mathrm{~s}$ time delay. In fact, Fig. 5_A shows that the frequency has a fast ramping at $\mathrm{t}=5.5 \mathrm{~s}$, which is an index of likely oscillations. The described behavior might be more perceptible from the ROCOF trend present in Fig. 5_B. It can be seen that the ROCOF arrives to have positive values in case of shortage of generation, in contrary with what described by the swing equation [13], [14] .

One can note that, large time delays lead to a less stable frequency behaviour. An explanation is that the controller
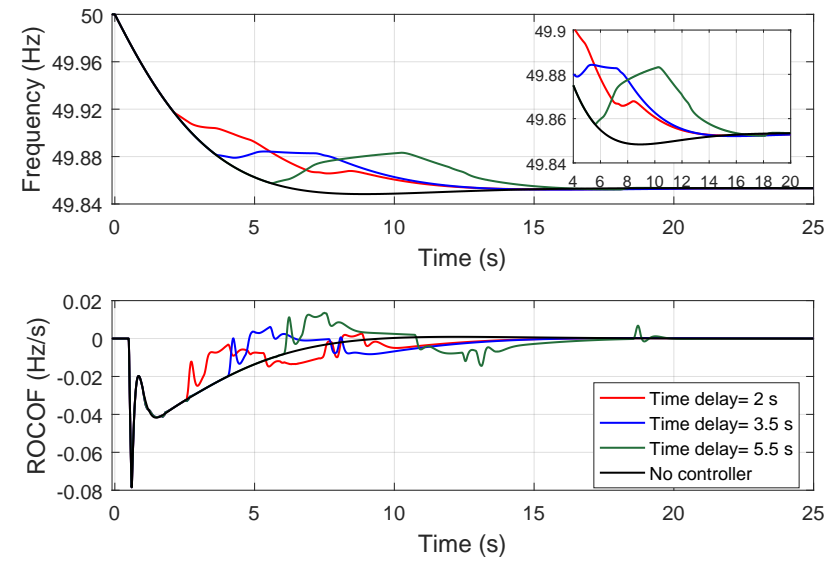

Fig. 5. Sensitivity Analysis, Fig. 5_A reports the frequency trends and Fig. 5_B reports the ROCOF trends.

performance is dependent from three parameters: System inertia, time delay and droop characteristic. The three parameters are strongly connected to each other. In fact, a high grid inertia allows the implementation of a steep droop, and employing a steep droop imposes the use of small time delay.

Since virtual inertia services must be delivered as fast as possible, and considering the results in this scenario, it has been decided to employ time delay of $2 \mathrm{~s}$ for Scenario 2 and Scenario 3.

\section{B. Scenario 2: Comparative analysis between VIC and VIC_S}

This scenario aims at evaluating the VIC and VIC_S effectiveness on the frequency dynamics, and at comparing the EV's current set-point. The frequency and ROCOF trends are shown in Fig. 6_A and Fig. 6_B, respectively. The VIC is represented with solid lines while VIC_S is represented with dashed lines.
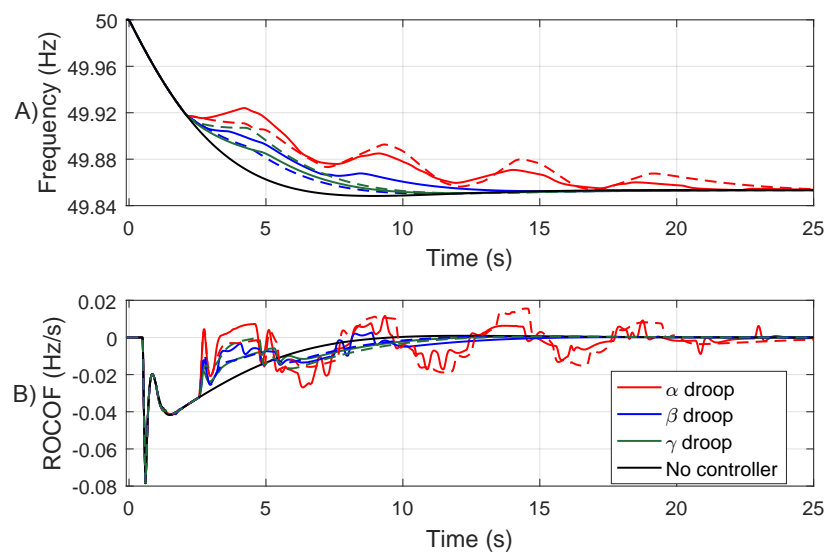

Fig. 6. Frequency and ROCOF trends employing VIC and VIC_S, Fig. 6_A reports the frequency trends and Fig. 6_B reports the ROCOF trends.

As shown in Fig. 6_A, the system frequency oscillates applying the $\alpha$ droop, and that is due to the combination of having a steep droop and relatively long time delay in terms of virtual inertia control (i.e., 2 s). The frequency oscillations 
could also be noticed from the ROCOF behavior presented in Fig. 6_B. In fact, also in this case the ROCOF begun to have positive values in the event of shortage of generation.

Due to the high inertia of the analysed grid, the two controllers, namely VIC and VIC_S have a similar effects on the frequency behaviour. On the other hand, from Table I, one can note that the number of switching between the EV's current set-point is substantially reduced by the VIC_S.

TABLE I

NUMBER OF SWITCHINGS

\begin{tabular}{||c|c|c||}
\hline Droop & VIC Nr. Switchings & VIC_S Nr. Switchings \\
\hline \hline$\alpha$ & 48 & 22 \\
\hline$\beta$ & 14 & 5 \\
\hline$\gamma$ & 6 & 3 \\
\hline
\end{tabular}

\section{Scenario 3: Performance analysis of VIC and VIC_S}

This scenario is composed by two study cases, the first one presents a comparative performance analysis between the two controllers in terms of frequency dynamics and EV's current oscillation. The second study case shows the advantages of applying a combined droop in case of VIC_S. Contrary to the previous scenario, the frequency is fluctuating around 50 $\mathrm{Hz}$ due to the wind generation. In the two study cases the system response is triggered by four load events as mentioned in section II-C.

1) Study case 1: ) The system is studied by using the three droops. The frequency and the ROCOF trends are presented in Fig. 7_A and Fig. 7_B, respectively. The VIC is represented with solid lines while VIC_S with dashed lines.
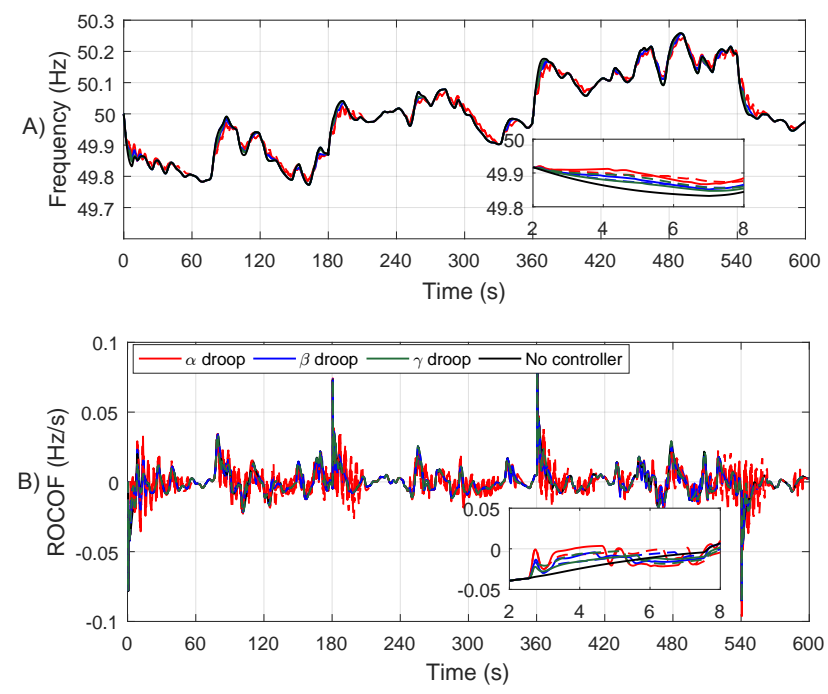

Fig. 7. Frequency and ROCOF trends employing VIC and VIC_S, Fig. 7_A reports the frequency trends and Fig. 7_B reports the ROCOF trends.

Fig. 7 shows the effectiveness of the VIC as well as VIC_S in reducing slightly the ROCOF compared to the non controlled case. It needs to be taken into account that this limited improvement is due the limited number of EVs participating in the control (i.e., three EVs). As explained in scenario 2, due to the high inertia of the modeled microgrid, the two controllers have a similar effects on the frequency behaviour. On the other hand, to highlight the higher performance of VIC_S compared to VIC on the EV's current set-point, the total number of switchings from one set-point to the other is reported in Table II.

TABLE II

NUMBER OF SWITCHINGS

\begin{tabular}{||c|c|c||}
\hline Droop & VIC Nr. Switchings & VIC_S Nr. Switchings \\
\hline \hline$\alpha$ & 528 & 300 \\
\hline$\beta$ & 120 & 38 \\
\hline$\gamma$ & 36 & 14 \\
\hline
\end{tabular}

As deducible from Table II, the VIC_S has reduced significantly the number of switchings operations for the different droops. The number of switchings has been reduced by $43 \%$, $68 \%$ and $61 \%$ for the $\alpha, \beta$ and $\gamma$ droop, respectively. This result is very valuable in the future perspective of integrating EVs for ancillary services (e.g., virtual inertia and frequency support). Since EVs will participate in the ancillary services during the whole charging process, they might be able to provide the same support performance with less degradation of the battery performances.

2) Study case 2: Generally speaking, the controller participation is proportional to the droop steepness. On one hand the controller should always participate with all the available power reserve to reduce the ROCOF. On the other hand, continuous regulation will result in reducing the reserves availability (i.e., the state of charge of the battery will be reduced).

To overcome this issue, it has been decided to impose a combined droop with a deadband. The idea is to avoid the controller participation in case of very small ROCOF by imposing a deadband (i.e., $\pm 0.01 \mathrm{~Hz} / \mathrm{s}$ ). Exceeded the deadband, to avoid the full participation of the controller, it has been applied a droop with the same slope as the $\beta$ droop, with a threshold at $\pm 0.035 \mathrm{~Hz} / \mathrm{s}$. To allow a higher participation of the controller in case of large events, exceeded the threshold, a droop with the same slope as the $\alpha$ droop will be applied, the ROCOF limits are $\pm 0.079 \mathrm{~Hz} / \mathrm{s}$. The implemented droop and the frequency behaviour are presented in Fig. 8 and Fig. 9 , respectively.

Contrary to the previous scenarios, the results show the very limited participation of the controller. This response presents an advantage in terms of EVs. In fact, since EVs are not dedicated to provide ancillary services, they can not be handled as the traditional reserves. On one hand, they must guarantee a certain state of charge for the end user. On the other hand, they must assure the reserve availability in case of large events. Fig. 9 shows that the controller participates in the regulation very few times, mainly in case of contingencies and thats due to the preselected droop. The desired participation rate could be achieved by combining different droops and/or different deadbands. 


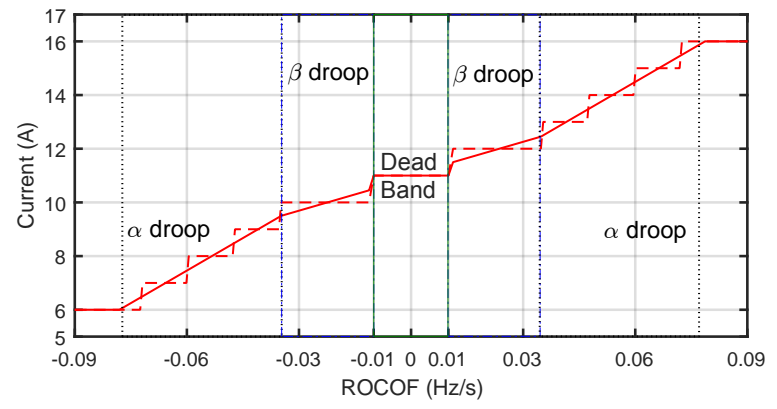

Fig. 8. Combined droop, composed by a deadband and the combination of $\alpha$ and $\beta$ droop.
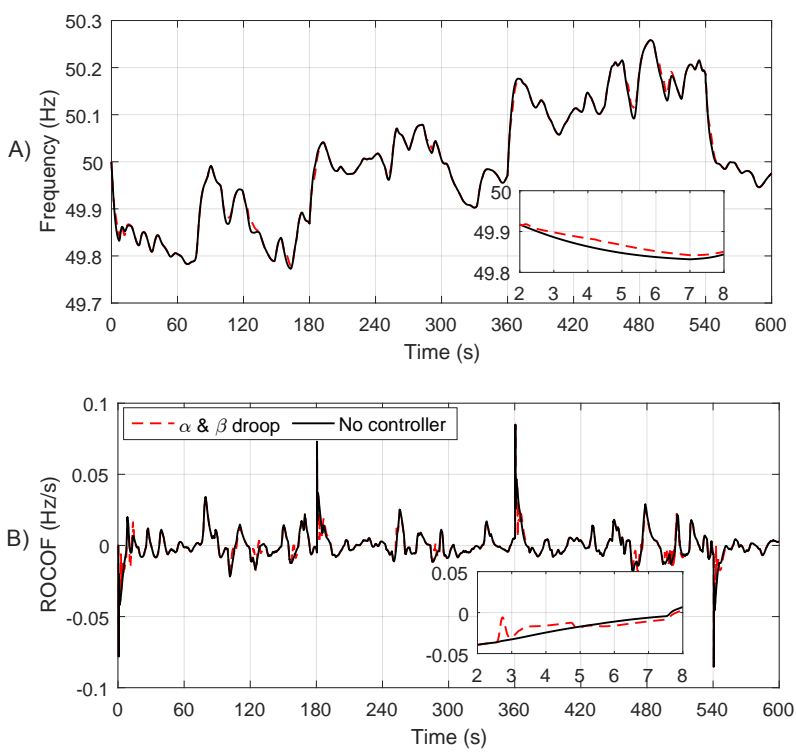

Fig. 9. Frequency and ROCOF trends employing VIC_S with a combined droop, Fig. 9_A reports the frequency trends and Fig. 9_B reports the ROCOF trends.

\section{CONCLUSION AND Future WORK}

The analysis showed the EVs capability of providing virtual inertia services and the time delay effect on the controller response and consequently on the frequency aspect. It was shown that the implementation of VIC employing EV might causes oscillations on the EVs current set-point. But It has also been demonstrated that the presented stabilizer algorithm was able to reduce the oscillations significantly.

It has been shown, that the controller can be applied, respecting the technical constraints imposed by the IEC 61851 standard, in a realistic situation with continuous frequency fluctuations due to wind generation.

Moreover, a combined droop has been applied in case of VIC_S to limit the controller participation in case of small ROCOF and allow a higher participation in case of large events.

Further analysis will be carried out to test the VIC and VIC_S developed controllers in the experimental facilities SYSLAB-
PowerLABDK. Future work will take into account the state of charge of the battery (SOC) in the developed controller to guaranty a certain SOC for the end user. Future work will also focus on modelling the battery considering the combination of the SOC and the implemented droop to represent the possible equivalent inertia of the battery.

\section{ACKNOWLEDGMENT}

Michel Rezkalla is Ph.D. student at the Technical University of Denmark (DTU) and is supported by the EU FP7 project ELECTRA (grant: 609687) and the Danish Research Project ELECTRA Top-up (grant: 3594756936313). More information at www.electrairp.eu

\section{REFERENCES}

[1] T. Ackermann, Wind Power in Power Systems. John Wiley, 2011.

[2] M. Marinelli, S. Massucco, A. Mansoldo, and M. Norton, "Analysis of Inertial Response and Primary Power-Frequency Control Provision by Doubly Fed Induction Generator Wind Turbines in a Small Power System," in 17th Power Systems Computation Conference, pp. 1-7, Aug 2011.

[3] P. W. Christensen and G. T. Tarnowski, "Inertia of wind power plants State-of-the-art review," in The 10th International Workshop on LargeScale of Wind Power, Aarhus, Denmark, 2011.

[4] E. Muljadi, V. Gevorgian, and M. Singh, "Understanding Inertial and Frequency Response of Wind Power Plants," in IEEE Symposium on Power Electronics and Machines in Wind Applications, pp. 1-8, IEEE Symposium on Power Electronics and Machines in Wind Applications, 2012.

[5] S. Sharma, S. H. Huang, and N. D. R. Sarma, "System inertial frequency response estimation and impact of renewable resources in ERCOT interconnection," IEEE Power and Energy Society General Meeting, pp. 1-6, 2011.

[6] J. H. Eto, "Use of Frequency Response Metrics to Assess the Planning and Operating Requirements for Reliable Integration of Variable Renewable Generation," tech. rep., Lawrence Berkeley National Laboratory, 2011.

[7] K. Clement-Nyns, E. Haesen, and J. Driesen, "The impact of charging plug-in hybrid electric vehicles on a residential distribution grid," IEEE Transactions on Power Systems, vol. 25, pp. 371-380, Feb 2010.

[8] M. Rezkalla, K. Heussen, M. Marinelli, J. Hu, and H. W. Bindner, "Identification of requirements for distribution management systems in the smart grid context," in Power Engineering Conference (UPEC), 2015 50th International Universities, pp. 1-6, Sept 2015.

[9] W. Kempton and J. Tomic, "Vehicle-to-grid power implementation: From stabilizing the grid to supporting large-scale renewable energy," Journal of Power Sources, J. Power Sources, J Power Sou, J Power Sources, vol. 144, no. 1, pp. 280-294, 2005.

[10] S. Martinenas, M. Marinelli, P. Andersen, and C. Traholt, "Implementation and demonstration of grid frequency support by $\mathrm{v} 2 \mathrm{~g}$ enabled electric vehicle," in Power Engineering Conference (UPEC), 2014 49th International Universities, pp. 1-6, Sept 2014.

[11] IEC 61851-1:2010, "Electric vehicle conductive charging system Part 1: General requirements," 2010.

[12] M. Marinelli, S. Martinenas, K. Knezović, and P. B. Andersen, "Validating a centralized approach to primary frequency control with series-produced electric vehicles," J. of Energy Storage, vol. 7, pp. 63-73, Aug. 2016.

[13] P. Kundur, Power System Stability and Control. McGraw-Hill, 1994.

[14] A. Ulbig, T. Borsche, and G. Andersson, Impact of Low Rotational Inertia on Power System Stability and Operation. The 19th World Congress of the International Federation of Automatic Control (IFAC14), Cape Town, South Africa, Aug. 2014. 